\title{
Proust et Hergé : de quelques points communs entre À la recherche du temps perdu et Les Aventures de Tintin
}

\author{
Première partie
}

SAMUEL BIDAUD

\begin{abstract}
Proust and Hergé: on some similarities between À la Recherche du temps perdu and Les Aventures de Tintin. Part I. Marcel Proust and Hergé seem to have nothing in common. Their works are indeed very different: they do not belong to the same genre, nor treat the same themes or have the same public. What parallel could be established between $A$ la Recherche $d u$ temps perdu (In Search of Lost Time), which revolutionized the genre of the novel, and Les Aventures de Tintin (The Adventures of Tintin), a series of comic albums apparently intended only for children? A closer study reveals however that Proust and Hergé, beyond what one could think at first sight, share deep similarities on which this article, published in two parts, will focus. First of all, $\grave{A}$ la Recherche du temps perdu as well as Les Aventures de Tintin rest on the creation of a specific world, which can be characterized by Balzac's principle of returning characters and by the importance of the imaginary of space (Proust's rêveries about the names of places, Hergé's fictitious geography). Moreover, Proust and Hergé's characters have a very singular language and linguistic features which can be identified easily (let us think of Dr. Cottard's puns, of Odette's anglicisms, etc. in Proust, or of Captain Haddock's insults or Dupond and Dupont's slips of the tongue in Hergé). Eventually, Proust and Hergé both develop a reflection on time which gives rise to a singular temporality in their books, and more precisely a reflection on lost and regained time, with two opposite situations and therefore two opposite conceptions for each of the authors. This first part of our study focuses on the principle of returning characters adopted by Proust and Hergé, on their imaginary of space and on the language of their characters, while the second part, which will be published in the next issue of Interlitteraria, will be devoted to the problematics of time.
\end{abstract}

Keywords: Marcel Proust; Hergé; À la Recherche du temps perdu; Les Aventures de Tintin; return of the characters; imaginary of space; characters' language; time and temporality 
A priori, rien ne rapproche Marcel Proust et Hergé : alors que le premier a révolutionné le genre romanesque au début du vingtième siècle, le second a longtemps été considéré (à tort) comme un simple auteur de bandes dessinées pour enfants; $A$ la Recherche du temps perdu est un roman que l'on peut qualifier d'essentiellement intérieur, Les Aventures de Tintin sont au contraire presque toujours tournées vers l'extérieur et vers l'action ; le narrateur de la Recherche, de santé fragile, ne s'aventure guère au-delà de Balbec et de Venise, Tintin au contraire voyage aux quatre coins du monde, et les oppositions des deux œuvres pourraient être multipliées. Pourquoi, alors, comparer ces dernières ? Certes, Hergé a lu Proust, mais on aurait bien des difficultés à repérer l'influence de celui-ci dans Les Aventures de Tintin. Pourtant, intuitivement, le lecteur a l'impression que les deux univers, celui de la Recherche et celui de Tintin, ont quelque chose en commun. Nous voyons au moins trois points qui permettent à la fois de rapprocher et confronter l'œuvre de Proust et celle d'Hergé : toutes deux reposent sur la création d'un monde spécifique, qui se caractérise par le principe balzacien du retour des personnages et par l'importance de l'imaginaire des lieux, les personnages se définissent par un langage très fortement marqué, et les deux œuvres s'inscrivent dans une conception singulière du temps.

Cette étude, pour des raisons de longueur, est publiée en deux parties. La première partie, qui correspond au texte qui suit, traite de la thématique des personnages récurrents, de l'imaginaire des lieux et du langage des personnages dans la Recherche et dans Tintin, alors que la seconde partie, à paraître dans le prochain numéro d'Interlitteraria de cette année, se penchera quant à elle sur la problématique du temps dans les œuvres de Proust et d'Hergé.

\section{La création d'un monde : personnages récurrents et imaginaire des lieux}

S'il est possible de parler, au sujet de l'œuvre de Proust comme de celle d'Hergé, de la création d'un véritable « monde », qui donne lieu à la publication de dictionnaires des personnages (voir par exemple, pour la Recherche, Erman 2010, et, pour Tintin, Mozgovine 1992), ainsi que, dans le cas de Proust, à un dictionnaire des lieux d'À la recherche du temps perdu (Erman 2011), c'est parce que les deux œuvres se caractérisent par le principe du retour des personnages et par la place qu'elles accordent à l'imaginaire des lieux, ce qui, comme nous allons le voir, contribue à en faire des univers à part entière. 
Proust et Hergé : de quelques points communs

\section{Le retour des personnages}

Ce que Proust admirait particulièrement chez Balzac, c'est, comme on le sait, la « trouvaille » de l'auteur de la Comédie humaine qui consista à faire réapparaître ses personnages d'un livre à l'autre : Balzac, « jetant sur ses ouvrages le regard à la fois d'un étranger et d'un père, [...] s'avisa brusquement en projetant sur eux une illumination rétrospective qu'ils seraient plus beaux réunis en un cycle où les mêmes personnages reviendraient et ajouta à son œuvre, en ce raccord, un coup de pinceau, le dernier et le plus sublime » (La Prisonnière, citée par CarrierLafleur 2010 : 239-240). Et Thomas Carrier-Lafleur commente (Ibid. 240) : « Cette unité, trouvée après coup, est pour le héros [le narrateur de la Recherche], futur homme de lettres, ce qu'il y a de plus réel et de plus beau. La vie, le sens d'une vie, ne fonctionne pas autrement $\gg$. Le retour des personnages fait bien sûr intervenir la question de la temporalité, et le personnage, chez Proust, est inséparable du temps dans lequel il s'inscrit. Les personnages paraissent à un moment, nous quittent à un autre, reviennent, à l'image de Gilberte que l'on connaît dans Du côté de chez Swann et À l'ombre des jeunes filles en fleurs et que l'on ne retrouve que dans Albertine disparue. Le retour des personnages, ou plutôt leur réapparition à intervalles irréguliers dans le roman, permet ainsi de développer la thématique du temps, et, surtout, des ravages de ce dernier, qui culminent dans la dernière scène du Temps retrouvé où le narrateur revoit, lors de la matinée Guermantes, tous les êtres qu'il a jadis fréquentés vieillis et méconnaissables, métamorphosés par le temps au point qu'ils lui font d'abord l'effet d'être déguisés :

Au premier moment je ne compris pas pourquoi j’hésitais à reconnaître le maître de maison, les invités, et pourquoi chacun semblait s'être «f fait une tête », généralement poudrée et qui les changeait complètement. Le prince avait encore en recevant cet air bonhomme d'un roi de féerie que je lui avais trouvé la première fois, mais cette fois, semblant s'être soumis lui-même à l'étiquette qu'il avait imposée à ses invités, il s'était affublé d'une barbe blanche et, traînant à ses pieds qu'elles alourdissaient comme des semelles de plomb, semblait avoir assumé de figurer un des « âges de la vie ». [...] À vrai dire je ne le reconnus qu'à l'aide d'un raisonnement et en concluant de la simple ressemblance de certains traits à une identité de la personne. (Proust, Le Temps retrouvé, 1999 : 2304) 
BIDAUD

Et le narrateur poursuit :

Je ne sais ce que le petit Fezensac avait mis sur sa figure, mais tandis que d'autres avaient blanchi, qui la moitié de leur barbe, qui leurs moustaches seulement, lui, sans s'embarrasser de ces teintures, avait trouvé le moyen de couvrir sa figure de rides, ses sourcils de poils hérissés, tout cela d'ailleurs ne lui seyait pas, son visage faisait l'effet d'être durci, bronzé, solennisé, cela le vieillissait tellement qu'on n'aurait plus dit du tout un jeune homme. Je fus bien plus étonné au même moment en entendant appeler duc de Châtellerault un petit vieillard aux moustaches argentées d'ambassadeur, dans lequel seul un petit bout de regard resté le même me permit de reconnaître le jeune homme que j'avais rencontré une fois en visite chez Mme de Villeparisis. (Ibid.)

Tous ou presque ont changé, de M. d'Argencourt à Legrandin, et, surtout, le narrateur lui-même, qui prend conscience, à travers les remarques qui lui sont faites, de sa propre vieillesse. Le retour des personnages contribue ainsi à faire prendre conscience du temps et de son passage, et contribue finalement en partie à l'accouchement de l'écriture du roman longtemps retardée, puisque c'est le temps qui constituera l'une des thématiques majeures de la Recherche:

Aussi, si elle [la force] m'était laissée assez longtemps pour accomplir mon œuvre, ne manquerais-je pas d'abord d'y décrire les hommes, cela dût-il les faire ressembler à des êtres monstrueux, comme occupant une place si considérable, à côté de celle si restreinte qui leur est réservée dans l'espace, une place au contraire prolongée sans mesure puisqu'ils touchent simultanément, comme des géants plongés dans les années à des époques, vécues par eux si distantes, entre lesquelles tant de jours sont venus se placer - dans le Temps. (Ibid. 2401)

Le retour des personnages est donc, chez Proust, intrinsèquement lié à la question du temps ; ce dernier a un effet destructeur sur les êtres, mais en même temps créateur sur le narrateur : le temps ne se donne à voir qu'à partir de la métamorphose physique des personnages, et c'est cette prise de conscience soudaine du temps qui est en même temps le déclencheur de l'écriture du roman proustien.

Le principe du retour des personnages a également été utilisé par Hergé, apparemment suite à la lecture d'un ouvrage de Félicien Marceau consacré à Balzac. Certes, Tintin et Milou étaient bien accompagnés au début des Dupondt, puis du capitaine Haddock, qui revenaient d'un album à l'autre, mais à partir des 7 boules de cristal commencent à réapparaître des personnages secondaires des 
précédents albums. C'est ainsi que l'on retrouve le général Alcazar, rencontré pour la première fois dans L'Oreille cassée où il était président du San Theodoros, et la Castafiore, rencontrée dans Le Sceptre d'Ottokar, au début des 7 boules de cristal, le premier ayant été renversé par une révolution et ayant émigré en Europe où il est devenu artiste de music-hall aux côtés de la seconde. Hergé adoptera progressivement par la suite le principe du retour des personnages : le docteur Müller de L'Île noire reparaît dans Au pays de l'or noir puis dans Coke en stock, Tchang, que Tintin sauve de la noyade dans Le Lotus bleu, est l'objet de la quête de l'aventure tibétaine dans Tintin au Tibet, Rastapopoulos, que le lecteur avait découvert dans Les Cigares $d u$ pharaon et dans sa suite immédiate Le Lotus bleu, revient dans Coke en stock et Vol 714 pour Sydney, etc. Certains personnages refont très souvent leur apparition à partir d'une époque : c'est le cas de la Castafiore et de Séraphin Lampion à partir de L'Affaire Tournesol. On peut noter que le retour des personnages est de plus en plus fréquent à partir de ce dernier album, ce qui correspond au moment où le rythme de publication des Aventures de Tintin ralentit d'une part, et où, d'autre part, Tintin ne recherche plus de lui-même l'aventure. Dans cette perspective, le retour des personnages signifie bien que l'univers de Tintin est stabilisé et qu'il n'y a plus de place pour le nouveau, à quelques exceptions près : seuls reviennent des personnages que l'on connaît déjà, et qui viennent confirmer (mais nous y reviendrons) une vision cyclique du temps. Ajoutons que Hergé joue également de ce retour des personnages d'un point de vue méta-littéraire : ainsi, lors du remaniement de ses albums à l'occasion de leur colorisation, il redessine discrètement les Dupondt sur la première case de Tintin au Congo alors qu'à l'origine les deux détectives n'apparaissaient pour la première fois que dans Les Cigares $d u$ pharaon, et il ajoute Allan dans Les Cigares du pharaon alors que ce dernier ne faisait son apparition que dans Le Crabe aux pinces d'or.

Le retour des personnages, qu'il s'agisse de celui de personnages secondaires qui deviennent presque des personnages principaux, à l'image de la Castafiore, ou de celui, ponctuel, de personnages secondaires qui restent secondaires, contribue à la création d'une véritable « famille de papier », pour reprendre une expression souvent utilisée au sujet des personnages de Tintin. Autour du noyau de base Tintin-Milou étendu aux Dupondt puis à Haddock et Tournesol, viennent ainsi graviter la Castafiore, Rastapopoulos et Séraphin Lampion, puis, au second plan, se détachent les Szut, Oliveira da Figueira ou Allan, et, enfin, à l'arrière-fond, les Dawson, Sakharine et autres, qui reviennent fugitivement comme pour rappeler leur existence le temps d'un album. Que le monde de Tintin constitue une famille, c'est ce que reflètent par ailleurs les doubles pages du début et de la fin des albums, où tous les personnages des Aventures sont 
BIDAUD

rassemblés dans des cadres. Comme le notait récemment Jean-Marie Embs à l'occasion de l'exposition Hergé au Grand Palais à Paris :

Hergé a disparu. Ses personnages restent. Les voici tous réunis devant nous sur cette fresque du Grand Palais. Alors, nous leur disons : Ainsi, vous êtes parfaits! Ne bougez surtout plus! Tenez la pose! Gardons-les tous intacts, tels qu'ils furent, réunis ici pour une photo de famille et une incessante renaissance dans nos têtes. (Embs $2016: 117$ )

\section{Imaginaire des lieux et lieux imaginaires}

La création d'un univers spécifique, dans À la recherche $d u$ temps perdu comme dans Les Aventures de Tintin, passe également par un imaginaire des lieux et par des lieux imaginaires : Combray ou Balbec chez Proust, la Syldavie, Moulinsart et bien d'autres chez Hergé.

On connaît l'importance des lieux dans $A ̀$ la recherche $d u$ temps perdu. Chez Proust, comme le note Michel Erman au début de l'ouvrage qu'il leur consacre, « les lieux ne figurent pas une toile de fond sur laquelle se déroulerait l'histoire : ils sont animés d'un génie qui retentit sur celle-ci » (Erman 2011 : 8). La Recherche tourne essentiellement autour de trois lieux : Combray, Balbec et Paris, seul ce dernier ayant une existence réelle. C'est assez dire que l'imaginaire du lieu est fondamental chez Proust. Combray et Balbec ont leur propre univers : Combray avec la maison de la tante Léonie ou la rivière de la Vivonne, Balbec avec son Grand Hôtel ou sa plage ; ils sont également situés par rapport à d'autres lieux, réels ou fictifs : on sait ainsi que Combray est situé non loin de Méséglise, et, dans Sodome et Gomorrhe, Brichot donne au narrateur dans le train qui les mène à La Raspelière l'étymologie d'un certain nombre de toponymes que l'on peut supposer situés non loin de Balbec. L'univers de la Recherche est ainsi un mélange de lieux réels et de lieux fictifs qui a sa propre cohérence. Mais le lieu, dans la Recherche, se présente avant tout comme un nom pour le narrateur, et, plus précisément, comme un nom fantasmé, d'où la possibilité de parler, littéralement, d'un «imaginaire des lieux ». La rêverie onomastique du narrateur regardant l'horaire des trains et les noms des villes où s'arrête « le train d'une heure vingt-deux » au début de « Noms de pays : le nom », la dernière partie de $\mathrm{Du}$ côté de chez Swann, est connue :

(M)ais j'avais beau les comparer [les noms des villes], comment choisir plus qu'entre des êtres individuels, qui ne sont pas interchangeables, entre Bayeux si haute dans sa noble dentelle rougeâtre et dont le faîte était illuminé par le 
Proust et Hergé : de quelques points communs

vieil or de sa dernière syllabe [...] ; Questambert, Pontorson, risibles et naïfs, plumes blanches et becs jaunes éparpillés sur la route de ces lieux fluviatiles et poétiques ; Benodet, nom à peine amarré que semble vouloir entraîner la rivière au milieu de ses algues, Pont-Aven, envolée blanche et rose de l'aile d'une coiffe légère qui se reflète en tremblant dans une eau verdie de canal ; Quimperlé, lui, mieux attaché et, depuis le moyen âge, entre les ruisseaux dont il gazouille et s'emperle en une grisaille pareille à celles que dessinent, à travers les toiles d'araignée d'une verrière, les rayons de soleil changés en pointes émoussées d'argent bruni ? (Proust, « Noms de pays : le nom », in Du côté de chez Swann, 1999 : 313)

Il en va de même avec Balbec, évoquée juste avant :

Quant à Balbec, c'était un de ces noms où comme sur une vieille poterie normande qui garde la couleur de la terre d'où elle fut tirée, on voit se peindre encore la représentation de quelque usage aboli, de quelque droit féodal, d'un état ancien de lieux, d'une manière désuète de prononcer qui en avait formé les syllabes hétéroclites et que je ne doutais pas de retrouver jusque chez l'aubergiste qui me servirait du café au lait à mon arrivée, me menant voir la mer déchaînée devant l'église et auquel je prêtais l'aspect disputeur, solennel et médiéval d'un personnage de fabliau. (Ibid.)

Le lieu n'est senti et vécu qu'à travers son nom, et sans aucun rapport avec la réalité. La confrontation avec cette dernière ne peut dès lors que s'avérer décevante : l'église de Balbec n'est pas telle que le narrateur l'avait imaginée à partir de ses rêveries onomastiques, et quelle déception qu'elle ne soit pas au bord de la mer mais à l'intérieur des terres ! Le lieu est ainsi, dans un premier temps, source de déception, et la Recherche, comme cela a été souligné, consiste d'ailleurs, selon les titres originellement envisagés par Proust pour deux des trois volumes qu'il prévoyait en 1913 (d'après Thierry Laget, cité par MarieAgnès Barathieu (2002: 64)), à passer de «l'âge des noms » à «l'âge des choses $\gg$, c'est-à-dire de l'illusion à la désillusion. Mais en même temps qu'il est vécu comme décevant, le lieu est également, dans un second temps, par le facteur du temps écoulé entre le moment où il a été vécu et le moment de la narration, revisité à travers le prisme du souvenir et de la mémoire involontaire et reparaît sous une forme nouvelle avec toutes les sensations dont le narrateur n'avait pas conscience alors qu'il s'y trouvait. On passe ainsi du lieu fantasmé au lieu réel et décevant, et du lieu réel et décevant au lieu métamorphosé par le temps et restitué au narrateur tel qu'il l'a ressenti alors sans s'en apercevoir, 
ce que l'écriture a finalement pour but de dire. Et c'est peut-être dans cet écart entre le nom, la réalité et le souvenir que réside l'essence du lieu proustien.

L'imaginaire des lieux est également fondamental chez Hergé, quoique sous une forme complètement différente. L'auteur des Aventures de Tintin a créé un monde, avec ses propres pays, qui, s'ils représentent une synthèse de pays réels, n'en sont pas moins imaginaires. Dès L'Oreille cassée apparaissent ainsi deux États fictifs situés en Amérique latine et qui sont en conflit constant, la République du San Theodoros et le Nuevo Rico. Tintin se rend essentiellement au San Theodoros et dans sa capitale Las Dopicos à deux reprises : la première fois dans L'Oreille cassée, et la seconde, une quarantaine d'années plus tard, dans Tintin et les Picaros. Le San Theodoros, en proie aux éternels renversements du général Alcazar et du général Tapioca qui se succèdent tour à tour au pouvoir, représente une caricature des dictatures d'Amérique latine, même si Hergé joue sur les stéréotypes dans Tintin et les Picaros, puisque, comme on n'a pas manqué de le relever, Alcazar, le communiste, est soutenu par l'International Banana Company (donc le capitalisme), alors que le général Tapioca, qui représente le capitalisme, est soutenu par la Bordurie, devenue communiste après Le Sceptre d'Ottokar. Autre pays imaginaire, le Khemed, dirigé par Ben Kalish Ezab, est une caricature des monarchies pétrolières ; il apparaît pour la première fois dans $A u$ pays de l'or noir, et Tintin s'y rend de nouveau dans Coke en stock.

C'est essentiellement la Syldavie, et son voisin, la Bordurie, qui retiendront notre attention, dans la mesure où c'est à ces deux pays qu'Hergé semble avoir accordé le plus d'importance. La Syldavie et la Bordurie, qui sont présents dans quatre albums (Le Sceptre d'Ottokar, Objectif Lune, On a marché sur la Lune et L'Affaire Tournesol), apparaissent pour la première fois dans Le Sceptre d'Ottokar, lequel, rappelons-le, raconte l'histoire d'un Anschluss manqué : la Bordurie, qui représente l'Allemagne de l'époque, tente d'annexer la Syldavie (dans laquelle beaucoup voient un symbole de la Belgique) en dérobant le sceptre du roi Muskar (qui fait songer au roi Léopold III), sans lequel ce dernier ne peut régner. Plusieurs chercheurs ont essayé, en vain, de situer la Syldavie, qui apparaît en réalité comme une synthèse des pays d'Europe de l'Est et des Balkans bien plus que comme un pays réel, comme c'est également le cas pour les autres pays inventés par Hergé (voir sur le sujet l'article que consacre Albert Algoud à la Syldavie dans son Dictionnaire amoureux de Tintin (2016: 624-632)). Ce qui frappe dans la représentation de la Syldavie, c'est sa vraisemblance. Le petit État nous est très précisément décrit dans un prospectus que lit Tintin dans l'avion qui le mène à Prague au début du Sceptre d'Ottokar et qui semble un véritable article encyclopédique : en 3 pages (19-21), Hergé dote la Syldavie d'une histoire, dont les premières traces remontent au $6^{\text {ème }}$ siècle avec l'arrivée des Slaves, d'une géographie, de costumes traditionnels, d'une architecture que l'on découvre 
dans une vue de la capitale du pays, Klow, d'une devise, et, à travers elle, d'une langue, le syldave, avec laquelle Tintin entre en contact peu après. Quant à la Bordurie, si elle était au début fortement inspirée des pays fascistes, elle devient, dans L'Affaire Tournesol, une caricature de la Russie soviétique, avec à sa tête le maréchal Plekszy-Gladz, dont les moustaches évoquent celles de Staline, le communisme ayant succédé au fascisme et une dictature en remplaçant une autre. Tous ces pays imaginaires mais très fortement vraisemblables constituent autant de lieux à découvrir pour Tintin, qui servent de décor au déroulement des aventures de ce dernier et de ses compagnons.

Le château de Moulinsart contraste avec les lieux précédents. Inspiré d'un château réel, celui de Cheverny, mais redessiné, le château de Moulinsart est acquis par le capitaine Haddock, comme on le sait, à la fin du Trésor de Rackham le Rouge, et est présent dans tous les albums qui suivent, exception faite de Tintin au Tibet et de Vol 714 pour Sydney d'une part, et, d'autre part, du Temple $d u$ soleil et de On a marché sur la Lune, qui, en tant que suites, respectivement des 7 boules de cristal et de Objectif Lune, commencent dès le début au Pérou et dans l'espace. Moulinsart apparaît donc 9 fois sur les 13 albums qui constituent la seconde partie de l'œuvre d'Hergé, ce qui en fait de loin le lieu le plus récurrent dans les Aventures de Tintin et confirme à la fois son importance et son aspect structurant. Moulinsart est aussi bien un lieu que l'on quitte qu'un lieu que l'on a hâte de retrouver, et, comme le note Pierre Fresnault-Deruelle (2007 : 90) :

Sauf exception [...], le château de Moulinsart, où règne l'obséquieux Nestor, n'est habité par le jeune reporter et le Capitaine qu'« entre » les aventures, en ces laps de temps où chute toute tension et où les héros reprennent leurs forces. Château des arrivées et des départs (au fil des albums, on assiste à un véritable florilège en la matière), Moulinsart est une sorte de coulisse suffisamment théâtrale pour que toute une mise en scène puisse s'y déployer.

Le château de Moulinsart, contrairement aux pays inventés par Hergé, est un lieu de stabilité pour Tintin, Haddock et Tournesol, qui s'y retrouvent après chaque voyage : si la Syldavie et les autres États imaginaires sont des lieux d'aventure vers lesquels les héros partent, Moulinsart, à l'inverse, est un lieu familier où Tintin et ses compagnons se reposent entre chaque aventure, et représente l'univers « domestique », pour reprendre l'adjectif qu'utilise Cyrille Mozgovine (2006 : 9) dans sa Préface aux Métamorphoses de Tintin de Jean-Marie Apostolidès, à côté de l'univers romanesque, en donnant à ce dernier adjectif son sens courant synonyme d'aventure. On pourra noter que l'apparition de Moulinsart coïncide plus ou moins avec l'adoption par Hergé du principe du retour des personnages et avec la constitution de la famille de Tintin au complet. En ce sens, Moulinsart 
BIDAUD

est lié à la deuxième phase de l'œuvre d'Hergé : celle où les personnages, ayant fait le tour du monde ou presque, n'ont plus rien à découvrir ni personne de nouveau à rencontrer, et forment une famille désormais au complet ou presque (seule manque la Castafiore).

\section{Le langage des personnages}

Benoît Peeters, dans une émission du 28 septembre 2016 diffusée sur France Culture et consacrée à Tintin, opposait deux types d'auteurs : ceux qui ont une langue plus ou moins « uniforme », qui « peut parfois être très belle » mais donne un ton similaire à tous leurs personnages, et ceux qui « colorent » leurs personnages par le langage. Il rapprochait dans cette perspective Proust et Hergé, qui ont tous deux un « goût des langages qui définissent un rôle, qui définissent un personnage $\gg$ : il y a un langage de Mme Verdurin ou du docteur Cottard comme il y a un langage des Dupondt ou de Séraphin Lampion, pour reprendre les exemples cités par Benoît Peeters. Et en effet, dans À la recherche $d u$ temps perdu comme dans Les Aventures de Tintin, les personnages ont un langage très fortement marqué.

Chez Proust, chaque personnage ou presque a des caractéristiques linguistiques typiques, a un «style » qui lui est propre : Cottard se caractérise par ses célèbres calembours, Brichot par ses discours alambiqués et ronflants, Odette par ses anglicismes, Françoise par son parler paysan qui rejoint en réalité celui de Mme de Guermantes par bien des aspects, le directeur du Grand Hôtel de Balbec par une confusion linguistique permanente, etc. Comme le notent par ailleurs Dorothée Baud-Butigieg et Alexandre Helque (2003 : 56) :

Certains personnages ne semblent devoir leur existence d'êtres de papier qu'à leurs caractéristiques langagières. C'est le cas de Mme Poussin, qui n’apparaît que brièvement dans le roman, et uniquement, semble-t-il, à cause de son utilisation incessante de l'expression « Tu m'en diras des nouvelles » [...].

Le langage est bien sûr à l'image de celui qui l'utilise : les mauvais calembours de Cottard reflètent la bêtise de ce dernier, la façon qu'a Brichot de s'exprimer reflète son pédantisme, etc. Le langage a également à première vue une fonction sociale, puisque, globalement, chez Proust, les domestiques s'expriment comme des domestiques, les bourgeois comme des bourgeois et les aristocrates comme des aristocrates : les premiers (Céleste et quelques autres mis à part) se caractérisent par une maîtrise plus ou moins approximative de la langue française, les seconds par le fait qu'ils adoptent toutes les expressions à la mode, 
ce qui reflète leur dynamisme et leur montée dans la société de l'époque, et les derniers par leur parler de coterie (l'esprit Guermantes, etc.), qui en fait un monde à part. Toutefois, comme l'a bien vu Sylvie Pierron (1999), le langage permet également de rapprocher des êtres que tout oppose a priori du point de vue social, comme Françoise et Mme de Guermantes, dont le parler conservateur, au niveau du lexique pour la première, de la prononciation pour la seconde, représente celui d'une époque passée où la servante et la duchesse se rejoignent. Ainsi, comme le note Sylvie Pierron, Françoise est comparée aux écrivains du dix-septième siècle par le narrateur, qui relève dans le second tome du Côté de Guermantes les tournures classiques qu'elle emploie, comme « faire réponse », à l'instar de Mme de Sévigné, ou son utilisation des verbes « plaindre » et « balancer » et du nom « ennui » dans le sens où les emploient respectivement La Bruyère, Saint-Simon et Corneille (d'après Pierron 1999 : 52-53). Quant à Mme de Guermantes, elle a gardé la vieille prononciation du français, d'où le rapprochement que fait le narrateur dans La Prisonnière (d'après Pierron ibid. 54) : « on retrouve le vieux langage et la vraie prononciation des mots avec une Mme de Guermantes ou une Françoise ».

Il y a enfin un comique de mots chez Proust, au sens large, depuis Odette qui parle anglais au narrateur pour ne pas être comprise d'autrui alors que tout le monde comprend l'anglais autour d'elle sauf justement le narrateur à qui elle s'adresse, au directeur du Grand Hôtel de Balbec dont le galimatias fondé sur le mélange de plusieurs langues donne lieu à des non-sens, en passant par le valet de pied de Françoise, Joseph Périgot, grand lecteur qui digère mal ce qu'il lit et qui, lorsqu'il écrit à sa famille ou aux gens de son village, utilise une langue littéraire qu'il ne comprend pas et qu'il mêle à une syntaxe fautive, d'où l'effet comique qui en résulte.

Le langage des personnages de Tintin est également très spécifique. Comme nous l'avons rappelé ailleurs (Bidaud 2017), dans l'imaginaire de milliers de lecteurs, le capitaine Haddock, c'est avant tout des jurons et des insultes qui lui sont personnels (« Mille millions de mille sabords ! », « Tonnerre de Brest ! », «Marin d'eau douce ! », « Moule à gaufre ! », etc. ; voir pour un recensement le Haddock illustré d'Albert Algoud (2004)) ; les Dupondt, c'est avant tout leur « Je dirais même plus » et leurs lapsus ; Tournesol se caractérise par sa surdité qui lui fait tout déformer ; la Castafiore, par le fait qu'elle est incapable d'appeler correctement son interlocuteur, surtout en ce qui concerne le capitaine Haddock dont elle déforme continuellement le nom, mais également en ce qui concerne Nestor qui devient Norbert ou Séraphin Lampion qui devient Lanterne, etc. Les personnages principaux ont donc chacun un style particulier, un idiolecte, qui permet de les identifier. 
Il y a, bien sûr, et c'est ce qui saute tout d'abord aux yeux, un très fort pouvoir comique du langage chez Hergé. On rit volontiers des lapsus des Dupondt ou des déformations de Tournesol. Mais le langage des personnages de Tintin correspond également à leur personnalité et aux thématiques qui leur sont liées. À leur personnalité : ainsi le langage de Tintin, par opposition à celui des autres personnages, se caractérise par sa neutralité ; il a une fonction essentiellement référentielle, et donne rarement lieu à des effets de comicité. Il rejoint en cela la personnalité de Tintin : à un personnage très peu marqué, aussi bien au niveau du caractère qu'au niveau physique avec son visage vide, correspond un langage neutre, qui a pour fonction de dire le monde de façon transparente. Du point de vue thématique, ensuite, le psittacisme des Dupondt reflète leur structure quasi gémellaire, puisque, à l'image de leurs noms qui ne se différencient orthographiquement que par un $\ll \mathrm{d} \gg$ final pour Dupond et $\mathrm{un} \ll \mathrm{t} \gg$ final pour Dupont, lorsque l'un des Dupondt veut surenchérir sur l'autre avec son « Je dirais même plus $\gg$, il répète ce qu'a dit le premier à un ou quelques phonèmes près, ce qui rejoint la thématique du (presque) double dont on sait l'importance chez Hergé. On peut d'ailleurs parler, chez les personnages principaux d'Hergé, à l'exception de Tintin, d'une utilisation pathologique du langage, ou plutôt d'une «psychopathologie » du langage, notamment en ce qui concerne Tournesol et les Dupondt, les multiples lapsus des deux détectives ayant d'ailleurs donné lieu à un relevé intégral et à une étude (Meyer 2007).

Si les personnages de Tintin ont un langage individuel très spécifique, il y a également, chez Hergé, une créativité linguistique que l'on ne retrouve pas chez Proust, puisque l'auteur des Aventures de Tintin « crée » plusieurs langues imaginaires : l'arumbaya, le bordure et, surtout, le syldave, la langue de la Syldavie. Cette dernière a déjà été abondamment étudiée et commentée (voir par exemple Justens et Préaux 2004, Grutman 2010, ainsi que les sites de Mark Rosenfelder et de Jean-Jacques De Gheyndt dont nous donnons les références dans la sitographie à la fin de cet article). Le syldave est en réalité fondé en grande partie sur le marollien de Bruxelles, parler dérivé du brabançon (dialecte néerlandais), auquel Hergé a donné une graphie slave, la Syldavie étant inspirée de l'Europe de l'Est ou balkanique, mais également et majoritairement une graphie latine, ce qui rappelle la situation du serbo-croate. On trouvera une description détaillée du syldave sur le site Hergé's Syldavian: A grammar de Mark Rosenfelder, qui en décrit la morphosyntaxe et le vocabulaire. Hergé a même doté le syldave d'une histoire, puisque, dans la brochure sur la Syldavie que lit Tintin dans Le Sceptre d'Ottokar, apparaît un vieux parchemin manuscrit. Le bordure, la langue de la Bordurie, l’État voisin de la Syldavie, est nettement moins documenté que le syldave, et la page Wikipédia consacrée à la Bordurie ne cite que onze mots en langue bordure. Jean-Jacques De Gheyndt remarque sur 
son site : «Le bordure - contexte historique oblige - est doté d'une consonance plus germanique que le syldave, visant à illustrer l'idéologie de ses locuteurs »; les deux langues sont toutefois très proches. Quant à l'arumbaya, langue parlée par les Indiens du San Theodoros, on peut remarquer qu'il a une structure nettement plus vocalique que le syldave et le bordure, afin de produire un effet mimétique des langues d'Amazonie telles qu’on les imagine généralement. On opposera par exemple le syldave : «Czesztot wzryzkar nietz on waghabontz !... » (Hergé, Le Sceptre d'Ottokar, [date d'édition non précisée] : 25) et l'arumbaya : « Nagoum wazenh !... Yommo !... Nagoum ennegang !... » (Hergé, Tintin et les Picaros, [date d'édition non précisée] : 34). L'arumbaya reste toutefois fondé, comme le rappelle Jean-Jacques De Gheyndt, sur le brabançon lui aussi.

\section{Conclusion}

Nous nous sommes penché dans ce qui précède sur plusieurs aspects qui permettent de rapprocher $\grave{A}$ la recherche du temps perdu et Les Aventures de Tintin: le principe du retour des personnages, la grande place accordée à l'imaginaire des lieux et le langage très caractérisé des personnages. Mais le traitement que font Proust et Hergé de ces thématiques diffère considérablement.

Si Proust et Hergé utilisent tous deux le principe du retour des personnages, celui-ci, toutefois, n'a pas la même fonction dans À la Recherche du temps perdu que dans Les Aventures de Tintin. Chez Proust les réapparitions des personnages, saisis à des étapes différentes du temps, donnent à voir le caractère destructeur de ce dernier dans toute sa profondeur, et sont en fin de compte, comme nous l'avons vu, à l'origine de l'écriture de la Recherche dans la mesure où c'est par l'écart entre ce que sont devenus les personnages et ce qu'ils ont été jadis que se fait jour la conscientisation du passage du temps, thématique majeure $\mathrm{du}$ roman à venir. Chez Hergé, au contraire, le retour des personnages contribue à en faire des êtres familiers qui forment une véritable famille dans la mémoire narrative des lecteurs et qui, par leur récurrence, viennent donner au temps une sorte de stabilité.

Plusieurs différences opposent également Proust et Hergé quant à la place occupée par l'imaginaire des lieux dans leurs œuvres. Chez Proust, le lieu a une fonction à la fois poétique, thématique et temporelle, et existe avant tout comme nom, c'est-à-dire sous forme de rêverie onomastique. On peut finalement pleinement parler d'un imaginaire des lieux, comme nous l'avons souligné, au sujet de la Recherche. À cet imaginaire des lieux, on peut opposer des lieux imaginaires chez Hergé. Ce qui frappe dans la géographie imaginaire hergéenne, c'est le degré de condensation d'États et de villes réels qui contribuent à la création de pays très fortement vraisemblables. Ce qui intéresse Hergé, à 
travers les pays qu'il invente, est de créer un arrière-fond vraisemblable, et les lieux imaginaires ont chez lui une fonction avant tout narrativo-descriptive qui contribue à l'«effet de réel », pour reprendre l'expression de Roland Barthes. Mais les lieux ont d'autre part une fonction structurante, et permettent d'opposer deux univers : celui de l'aventure avec la Syldavie, la Bordurie et autres pays imaginaires, et celui, domestique, de Moulinsart, qui devient de plus en plus présent dans la seconde partie des Aventures de Tintin.

Les personnages d'À la recherche du temps perdu et des Aventures de Tintin, enfin, ont un langage fortement individualisé, qui assure, chez Proust et Hergé, des fonctions variées, d'une fonction comique à une fonction thématique. La différence essentielle entre Proust et Hergé est que, chez le premier, tous les personnages partagent le même code, qu'ils actualisent de façons variées, alors que chez le second, au contraire, plusieurs langues cohabitent, dans une proportion toute relative bien sûr puisque, à quelques exceptions près, c'est presque toujours le français qui sert de langue de communication, à côté toutefois du syldave, du bordure ou de l'arumbaya. Ces langues imaginaires, qui sont dotées d'une structure complexe, contribuent donc là encore à l'« effet de réel $\gg$ hergéen.

Il nous reste désormais à étudier, dans la seconde partie à paraître de cet article, la thématique du temps dans À la recherche $d u$ temps perdu et Les Aventures de Tintin.

\author{
Samuel Bidaud \\ bidaudsamuel@gmail.com \\ Univerzita Palackého v Olomouci \\ Neředín 4,804 \\ U Letiště 847 \\ 77900 Olomouc \\ ČESKÁ REPUBLIKA / RÉPUBLIQUE TCHÈQUE
}

\title{
Bibliographie
}

Algoud, A. 2004. Le Haddock illustré. Bruxelles-Paris : Casterman.

Algoud, A. 2016. Dictionnaire amoureux de Tintin. Paris : Plon.

Apostolidès, J.-M. 2006. Les métamorphoses de Tintin. Paris : Flammarion.

Barathieu, M.-A. 2002. Les mobiles de Marcel Proust. Une sémantique du déplacement. Villeneuve-d'Ascq : Presses Universitaires du Septentrion.

Baud-Butigieg, D., Helque, A. 2003. Proust. Levallois-Perret : Jeunes éd. - Studyrama. Bidaud, S. 2017. Tintin et le langage. - Estudios románicos, 26, 143-158.

Carrier-Lafleur, T. 2011. Introspection créatrice et comédie humaine. Proust, Balzac et Bergson. - @ nalyses, 6/3, 235-277. 
Proust et Hergé : de quelques points communs

Embs, J.-M. 2016. Une famille de papier. La foire aux solitudes. - Collectif, Hergé, catalogue de l'exposition au Grand Palais. Paris : Réunion des Musées NationauxGrand Palais/Bruxelles : Éditions Moulinsart, 111-139.

Erman, M. 2010. Le Bottin proustien. Qui est qui dans la Recherche ?. Paris : Éditions de la Table ronde.

Erman, M. 2011. Le Bottin des lieux proustiens. Paris : Éditions de la Table ronde.

Fresnault-Deruelle, P. 2007. Moulinsart, demeure aspirante et refoulante. - MEI, 27, $89-96$.

Grutman, R. 2010. « Eih bennek, eih blavek » : l'inscription du bruxellois dans Le Sceptre d'Ottokar. - Études françaises, 46(2), 83-99.

Justens, D., Préaux, A. 2004. Tintin : ketje de Bruxelles. Paris : Casterman.

Meyer, J.-P. 2007. Étude d'un corpus particulier de perturbation langagière : les lapsus de Dupond et Dupont dans les “Aventures de Tintin” (Hergé). - B. Vaxelaire, R. Sock, G. Kleiber, F. Marsac, éds., Perturbations et Réajustements. Langue et Langage. Strasbourg : Publications de l’Université Marc-Bloch, 297-310.

Mozgovine, C. 1992. Dictionnaire des noms propres de Tintin : de Abdallah à Zorrino. Préface de A. Algoud. Paris : Casterman.

Mozgovine, C. 2006. La stabilité des métamorphoses. Préface à Les métamorphoses de Tintin de J.-M. Apostolidès. Paris : Flammarion, 7-10.

Pierron, S. 1999. La « langue Françoise » dans À la recherche du temps perdu. Littérature, 116/4, 47-58.

\section{Euvres citées}

Hergé1. [1931]. Tintin au Congo. Tournai : Casterman. [Première publication aux Éditions du Petit Vingtième]

Hergé. [1934]. Les Cigares du pharaon. Tournai : Casterman.

Hergé. [1936]. Le Lotus bleu. Tournai : Casterman.

Hergé. [1937]. L'Oreille cassée. Tournai : Casterman.

Hergé. [1938]. L'Île noire. Tournai : Casterman.

Hergé. [1939]. Le Sceptre d'Ottokar. Tournai : Casterman.

Hergé. [1941]. Le Crabe aux pinces d'or. Tournai : Casterman.

Hergé. [1944]. Le Trésor de Rackham le Rouge. Tournai : Casterman.

Hergé. [1948]. Les 7 boules de cristal. Tournai : Casterman.

Hergé. [1949]. Le Temple du soleil. Tournai : Casterman.

Hergé. [1950]. Tintin au pays de l'or noir. Tournai : Casterman.

Hergé. [1953]. Objectif Lune. Tournai : Casterman.

Hergé. [1954]. On a marché sur la Lune. Tournai : Casterman.

1 Les albums auxquels nous avons fait référence dans l'article ne portent pas de date d'édition ; nous les citons donc dans l'ordre chronologique de leur parution, en mentionnant entre crochets la date de la première publication. Nous ne tenons pas compte du remaniement des albums et suivons l'ordre chronologique dans lequel ces derniers sont présentés sur la quatrième de couverture des Aventures de Tintin. 
BIDAUD

Hergé. [1956]. L'Affaire Tournesol. Tournai : Casterman.

Hergé. [1958]. Coke en stock. Tournai : Casterman.

Hergé. [1960]. Tintin au Tibet. Tournai : Casterman.

Hergé. [1968]. Vol 714 pour Sydney. Tournai : Casterman.

Hergé. [1976]. Tintin et les Picaros. Tournai : Casterman.

Proust, M. 1999. À la recherche du temps perdu. Paris : Gallimard. [Cette édition réunit les 8 volumes de la Recherche]

\section{Sitographie}

De Gheyndt, J.-J. Pour la Science et pour la Zwanze. Disponible sur <www.sciencezwanze.be/413830204> [Dernière consultation en juillet 2017]

Rosenfelder, M. Hergé's Syldavian: A grammar. Disponible sur < www.zompist.com/ syldavian.html $>$ [Dernière consultation en juillet 2017]

Wikipédia. Bordurie. Disponible sur <https://fr.wikipedia.org/wiki/Bordurie\# Bordurie_et_Syldavie $>$ [Dernière consultation en juillet 2017]

\section{Émissions radiophoniques}

Peeters, B. 28 septembre 2016. Intervention dans l'émission Le capitaine Haddock et les Dupondt : à chaque personnage son langage. - France Culture. Disponible sur $<$ https://www.franceculture.fr/bd/le-capitaine-haddock-et-les-dupondt-chaquepersonnage-son-langage $>$ [Dernière consultation en juillet 2017] 